\title{
Goal-driven modulation of oculomotor capture
}

\author{
CASIMIR J. H. LUDWIG and IAIN D. GILCHRIST \\ University of Bristol, Bristol, England
}

\begin{abstract}
In a recent study, Ludwig and Gilchrist (2002) showed that stimulus-driven oculomotor capture by abrupt onset distractors was modulated by distractor-target similarity: Participants were more likely to fixate an irrelevant onset when it shared the target color. Here we test whether this pattern of performance is the result of (1) inhibition of all items in the distractor color, (2) a response bias to local color discontinuities, or (3) the integration of stimulus-driven abrupt onset signals with goal-driven information about the target features. The results of two experiments clearly support the third explanation. We conclude that oculomotor capture is modulated by, but not contingent upon, top-down control, and our findings argue for an integrative view of the saccadic system.
\end{abstract}

Confronted with dynamic visual environments in which items appear, disappear, move, and change, it is essential to effectively select the behaviorally relevant portions of the visual field for more detailed processing. What is selected is determined by properties of the visual scene, on the one hand, and by the goals and intentions of the observer, on the other. The control of visual attention by both stimulus-driven and goal-driven factors has been studied extensively over the last two decades (for a review, see Egeth \& Yantis, 1997).

The potential to capture attention in a stimulus-driven way is particularly well established for new items that appear in the visual scene with an abrupt onset. This has typically been shown in visual search studies in which the time taken to find the target among a set of distractors indicates the efficiency of selection. In searches of arrays of no-onset elements for a specific letter (Enns, Austen, Di Lollo, Rauschenberger, \& Yantis, 2001; Yantis \& Jonides, 1984, 1990) or for an item of a specific color (Theeuwes, 1994), the appearance of an abrupt onset distractor is associated with a cost in target detection time. These findings have recently been extended into the oculomotor domain (Irwin, Colcombe, Kramer, \& Hahn, 2000; Theeuwes, Kramer, Hahn, \& Irwin, 1998; Theeuwes, Kramer, Hahn, Irwin, \& Zelinsky, 1999): Observers frequently made an erroneous saccade to the abrupt onset distractor before fixating the target.

However, in a series of attentional cuing studies, Folk, Remington, and colleagues (Folk, Remington, \& Johnston, 1992; Folk, Remington, \& Wright, 1994) have shown the importance of goal-driven attentional control

This work was supported by a University of Bristol postgraduate research scholarship awarded to C.J.H.L, and by a grant from the Wellcome Trust to I.D.G. We thank Kim Shapiro, Robert Rauschenberger, and an anonymous reviewer for their helpful suggestions on an earlier version of this article. Correspondence should be addressed to C. J. H. Ludwig, Department of Experimental Psychology, University of Bristol, 8 Woodland Road, Bristol BS8 1TN, England (e-mail: c.ludwig@ bristol.ac.uk). settings in mediating capture. In their paradigm, the target display is preceded by either a valid or an invalid spatial cue. A cost in the latency of manual responses to a target that is presented in a different location than the cue (i.e., a validity effect) is taken as evidence for attentional capture. Such a validity effect was obtained only when the cue and the target shared a relevant feature. For instance, responses to a color target were slowed down when preceded by an invalid color cue, but not by an invalid abrupt onset cue. The contingent capture hypothesis states that capture is never purely stimulus driven, and ultimately depends on the visual features the attentional system has been set up to respond to.

Evidence for stimulus-driven capture has come mainly from visual search studies, whereas the contingent capture hypothesis has received support mainly from attentional cuing studies. In a recent article, Ludwig and Gilchrist (2002) assessed the contribution of goal-driven control settings to stimulus-driven capture by abrupt onsets in a typical visual search paradigm. Because this paradigm served as the basis for the two experiments reported here, it will be described in greater detail below.

The task was to signal the location of a red no-onset target among three green no-onset distractors. These items were distributed over four locations, arranged in an imaginary rectangle centered on fixation. The possible target locations were indicated by gray placeholders, of similar size and luminance as the subsequent search elements. Having the search display preceded by such a placeholder display is an effective way of creating noonset search elements: The search array is created by simply changing the chromaticity of the placeholders. On a proportion of trials, an additional distractor appeared simultaneously with the search display in one of two locations, left or right of the center on the horizontal midline. This additional item could either have an abrupt onset or not, and could be similar (identical) or dissimilar (but identical to the other distractors) to the target. In separate blocks the target location was indicated either 
manually or with a saccade, but for the present purposes only the eye movement data will be considered. Schematic examples of the different display types are given in Figure 1, with some illustrative data from 1 observer.

We reasoned that if capture were entirely contingent upon attentional control settings, only the additional distractors that were similar to the target should disrupt the target-directed responses. Whether or not this distractor had an abrupt onset should not matter: The luminance increments could not be used to locate the target and the attentional system should not have been set up to respond to this particular feature. Similar distractors did indeed disrupt saccade programming more than the dissimilar ones. However, capture was particularly pronounced when the similar distractor had an abrupt onset. In addition, capture occurred on a small number of trials with dissimilar onset distractors. This pattern of results is clearly problematic for any strong version of the contingent capture hypothesis.

This modulation of oculomotor capture by target similarity also has implications for theories and models of saccade programming. An idea that is highly prevalent in the oculomotor literature is that of distinct neural pathways for different types of saccades (see, e.g., Gaymard, Ploner, Rivaud, Vermersch, \& Pierrot-Deseilligny, 1998; Guitton, Butchel, \& Douglas, 1985): stimulus-driven or "reflexive" saccades triggered by a sudden luminance onset in the visual field, and more goal-driven or "voluntary" saccades. The former type of eye movements is thought to be mediated by a fast route from striate cortex and parietal eye fields (PEF) into the superior colliculus (SC), a midbrain structure important for saccade generation (parieto-tectal route). The goal-driven movements are assumed to depend on the frontal eye fields (FEF) and its projections to the SC and the brainstem saccade generator. Theeuwes and his colleagues (Theeuwes, Kramer, Hahn, \& Irwin, 1998; Theeuwes et al., 1999) explained oculomotor capture in terms of a race between a stimulusdriven saccade program associated with the abrupt onset distractor and a goal-driven program for the correct targetdirected saccade.

The modulation of capture by color similarity seems inconsistent with this scheme. If capture by irrelevant onsets is mediated by the parieto-tectal pathway, this system must have been set up to respond to transients of a particular color. This is not likely, given that the projections from V1/PEF into the SC are predominantly magnocellular and thus insensitive to chromatic identity (Schiller, 1996). Because both similar and dissimilar onsets had the same (high) luminance contrast, one would not expect the color of an abrupt onset to make such a large difference. Our results suggest that abrupt onset (M-type) and target similarity (P-type, in this particular case) signals are integrated in a common saccade programming map (Croner \& Albright, 1999).

However, it is crucial to establish that increased oculomotor capture by irrelevant onsets that are similar to the target is in fact a modulation by target similarity and not the result of alternative top-down mechanisms that participants could have used to locate the target. This is the aim of the present paper. First, note that in the three experiments reported by Ludwig and Gilchrist (2002), the target and distractor colors were kept constant throughout. This may have enabled observers to inhibit all elements in the distractor color, attenuating capture by the dissimilar onsets. The contribution of this strategy is explored in Experiment 1, in which search for a known target is compared with search for a color singleton tar-

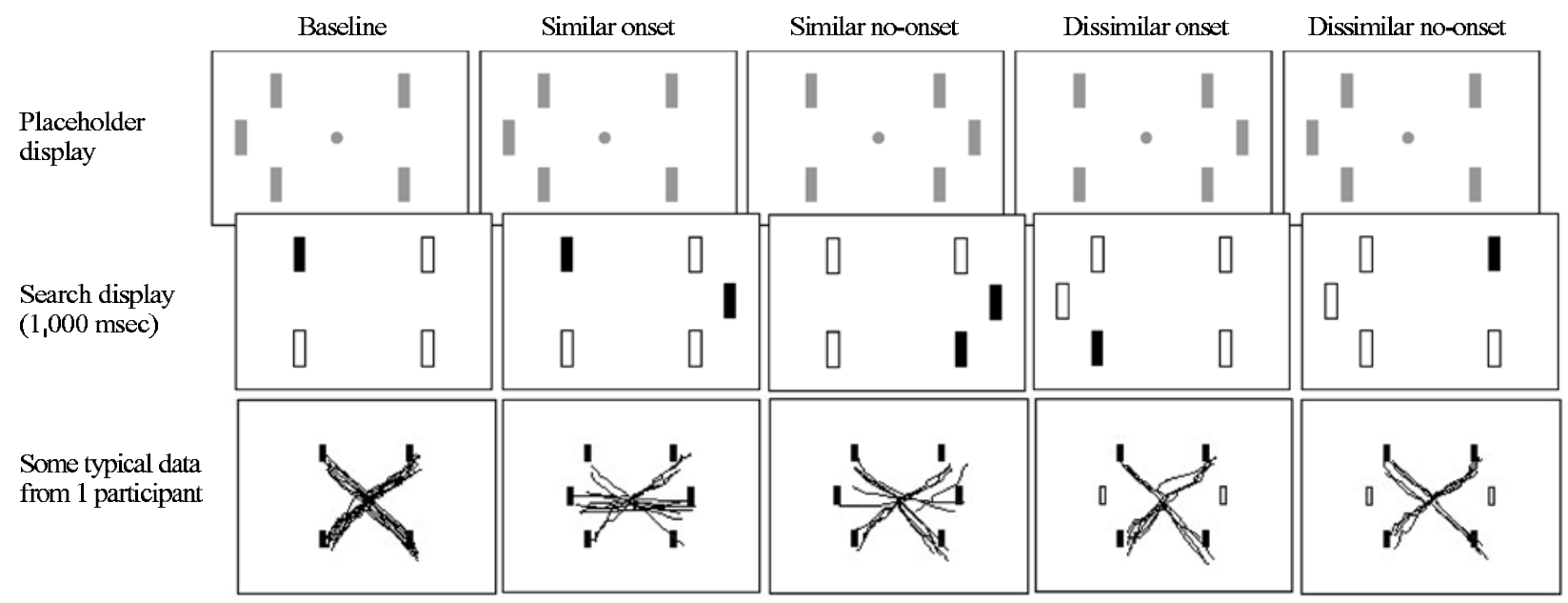

Figure 1. Schematic illustration of the sequence and timing of events in the five different trial types of Experiment 2 in Ludwig and Gilchrist (2002). Placeholders are indicated by the gray bars, bars in a distractor color are white, and bars in the target color are black. The bottom panels show the first saccade traces from 1 participant. These data were fairly typical for the overall pattern of results. The two experiments reported here used the same paradigm. 
get. In the latter condition, target and distractor colors varied unpredictably, and the strategy of inhibiting all items in the distractor color was therefore unavailable.

Second, the target was always of a different color than most of its surrounding elements, and on the majority of trials it was in fact the only item of a different color. Therefore, observers may have responded not to the target color itself, but instead to local color discontinuities to locate the target, enhancing capture by similar onsets. Experiment 2 investigated the possible contribution of this strategy by presenting additional distractors that are dissimilar from the target but that are also different from the distractors and therefore do create a color discontinuity (see Bacon \& Egeth, 1994).

\section{EXPERIMENT 1}

Participants were required to saccade to a color target and told to ignore additional distractors that appeared either with or without an abrupt onset. In two separate "constant target blocks," the target was either red or green; these blocks form a direct replication of the conditions of Experiment 2 in Ludwig and Gilchrist (2002). In an additional block, the target could be either red or green, and varied unpredictably from trial to trial (i.e., the target was a color singleton). If dissimilar onsets were not as disruptive as similar ones because participants inhibited all items in the distractor color, interference from dissimilar onsets should increase in the color singleton block.

\section{Method}

Participants. Twelve observers (6 men and 6 women; age range 18-28 years) took part for course credit or payment. All had selfreported normal or corrected-to-normal vision and were able to discriminate the colors used in this experiment.

Equipment and Stimuli. The typical display sequences, in the different trials of a constant target block, are illustrated in Figure 1. Placeholder displays consisted of a central gray circular disk (with a radius of $0.3^{\circ}$ ) that served as the fixation point and four gray vertical bars subtending $0.6^{\circ} \times 1.9^{\circ}$ indicating the possible target locations. A fifth placeholder was added in one of the two distractor locations, either left or right on the horizontal midline. This additional placeholder provided no information about whether or where a given type of distractor would be presented. Thus, on no-distractor baseline trials it would simply disappear, on trials with an additional onset distractor it would disappear and the distractor would appear on the other side of fixation, and on trials with an additional noonset distractor the placeholder would change color (just like the other four). ${ }^{1}$ Placeholders and fixation point were of the same chromaticity (CIE $x, y$ chromaticity coordinates of .28/.30) and luminance $\left(7.6 \mathrm{~cd} / \mathrm{m}^{2}\right)$, and were presented on a black background $\left(0.0 \mathrm{~cd} / \mathrm{m}^{2}\right)$. The four placeholders that indicated the possible target locations formed an imaginary rectangle centered on fixation. With the two distractor locations, there were six positions in which elements could be presented; these locations were arranged on the circumference of an imaginary circle around fixation with a radius of $7.3^{\circ}$. The search displays consisted of red (CIE coordinates of $.63 / .33$ ) and green (CIE coordinates of .29/.59) vertical bars of the same size and similar luminance as the placeholders.

Displays were presented on a 17 -in. SVGA monitor with $800 \times$ 600 pixel resolution and $74-\mathrm{Hz}$ refresh rate. The monitor was lo- cated at $57 \mathrm{~cm}$ from a chinrest. A second PC was used to record eye position data on-line. Eye movements were monitored at $250 \mathrm{~Hz}$ with the SMI EyeLink System (SensoMotoric Instruments GmbH, Teltow, Germany). Saccade onset was defined as a change in eye position with a minimum velocity of $35 \% \mathrm{sec}$ or a minimum acceleration of $9,500^{\circ} / \mathrm{sec}^{2}$.

Procedure. Each block of trials started with a nine-point grid calibration and validation procedure. Participants were asked to fixate a gray circular disk (identical to the fixation point) that appeared sequentially (but unpredictably) in a $3 \times 3$ grid. After a satisfactory validation had been obtained, a block of trials was run. Observers performed three blocks in which they had to saccade to the target as quickly as possible. There were two constant target blocks: In one block, participants searched for a red target among green distractors and in another block the target and distractor colors were reversed. In a third block, the target was a color singleton and varied unpredictably.

Each block had 106 trials, the first 10 of which were practice. In the constant target blocks, the remaining 96 trials were distributed as follows: One-third of the trials had no additional distractor (i.e., baseline trials), one-third were onset trials (half of which were similar to the target), and one-third were no-onset trials (again, half of which were similar). The trials with a similar distractor were dropped in the color singleton block because otherwise the target would no longer have been a color singleton. The three remaining trial types were distributed equally over the 96 trials. On half of the trials, the target was red and distractors were green; target and distractor colors were reversed in the other half of the trials. Additional distractors were presented equally often on the left and right of fixation. In all three blocks the different trial types were intermixed randomly. The order in which the blocks were run was counterbalanced across participants.

Data analysis. Trials on which eye position deviated more than $2^{\circ}$ from the central fixation point were rejected. The same applied to trials with anticipatory eye movements (latency less than $125 \mathrm{msec}$ ) and saccades with an amplitude smaller than $2^{\circ}$ or larger than $12^{\circ}$.

The remaining saccades were classified as having landed either on the target, on the additional distractor, on any of the other distractors, or in an empty display segment. Data from the two constant target blocks were analyzed together; the results from the color singleton block were dealt with separately. The main variable of interest was the accuracy of the first saccade - that is, the percentage of first saccades that landed directly on the target. Because in some conditions this percentage fell near the upper endpoint of the scale, the numbers were transformed into corrected arc sine values to approach a normal distribution (Milligan, 1987). These transformed values were then submitted to a repeated measures analysis of variance (ANOVA) with target color (red/green), similarity (similar/ dissimilar), and onset (onset/no-onset) as the three factors. The percentage target-directed saccades in the color singleton block was analyzed using the Wilcoxon signed rank test. This test was also used for the planned comparisons between conditions (across different blocks). The latencies of saccades to the target and to the additional distractor are also reported. Because the additional distractor did not capture the eyes very often in some conditions, the incorrect latencies were not analyzed in greater detail.

\section{Results and Discussion}

Of all trials, $2.11 \%$ were rejected due to improper fixation, $6.37 \%$ as a result of too small or too large a saccade, and $1.19 \%$ because of anticipatory responses. In the two constant target blocks, the vast majority of the eye movements landed on either the target or on the additional distractor. Pooled over the different display 
Table 1

Mean Percentage of First Saccades Directed to the Additional Distractor in the Three Blocks of Experiment 1

\begin{tabular}{|c|c|c|c|c|}
\hline \multirow[b]{2}{*}{ Target Type } & \multicolumn{4}{|c|}{ Distractor Type } \\
\hline & $\begin{array}{c}\text { Similar } \\
\text { Onset }\end{array}$ & $\begin{array}{c}\text { Similar } \\
\text { No-Onset }\end{array}$ & $\begin{array}{c}\text { Dissimilar } \\
\text { Onset }\end{array}$ & $\begin{array}{l}\text { Dissimilar } \\
\text { No-Onset }\end{array}$ \\
\hline Red & 45 & 25 & 3 & 0 \\
\hline Green & 39 & 22 & 1 & 0 \\
\hline Singleton & - & - & 9 & 1 \\
\hline
\end{tabular}

Note-No data were collected for cells containing a dash.

types, only $1.65 \%$ of all first saccades landed on another distractor or in an empty region of the display. Because this number is so small, these saccades were omitted from further analyses. The reported percentages of erroneous first saccades were based on just the target-directed saccades and those to the additional distractor. This procedure was also followed in Ludwig and Gilchrist (2002).

Table 1 lists the mean percentage of first saccades to the additional distractor in all three blocks. The ANOVA on the accuracy data showed no main effect of target color, and this factor did not interact with any of the other factors. This result is important because it suggests that the strong oculomotor capture observed for red onsets in previous experiments (Ludwig \& Gilchrist, 2002) was not due to possible subtle differences in luminance between the red and green distractors. In fact, regardless of the target color, the pattern of performance in the two constant target blocks replicated that observed in our previous experiments. That is, there were main effects of both onset and similarity $[F(1,11)=10.78, p<.05$, and $F(1,11)=57.02, p<.01$, respectively $]$. The interaction between both factors also reached significance $[F(1,11)=$ $8.04, p<.05]$, indicating that the effect of onset is larger for the similar distractors than for the dissimilar ones. However, given the small number of saccades to the dissimilar distractors, the functional importance of this interaction is unclear.

In the color singleton block, a small percentage (2.07\%) of first saccades landed in an empty segment. Search was more difficult than when the target and distractor colors were kept constant, as evidenced by a larger per- centage of first saccades directed to distractors other than the additional one $(9.94 \%, 5.88 \%$, and $5.93 \%$ in baseline, onset, and no-onset trials, respectively). Because these numbers did not differ between the onset and no-onset trial types $[T(10)=5.33, p>.05]$, those trials were excluded from the analysis below. Direct comparison of the accuracy in onset and no-onset trials showed that dissimilar onset distractors elicited more erroneous eye movements than the dissimilar no-onset distractors $[T(10)=0.00, p<.05]$. Planned comparisons showed that the same was true in the constant target blocks $[T(6)=1.00, p<.05]$, but that the dissimilar onsets in the color singleton block were more disruptive than dissimilar onsets in the constant target blocks $[T(11)=2.50, p<$ $.01]$. Not being able to inhibit items in the distractor color increased the interference from dissimilar onset distractors, yet still not to the extent of similar onset distractors $[T(12)=0.00, p<.01]$.

The mean latencies of first saccades to the target and to the additional distractor can be found in Table 2 . The mean latencies of the correct saccades in the constant target blocks were submitted to the same ANOVA as the accuracy data. Latencies were longer in the presence of a similar distractor, irrespective of whether this had an abrupt onset or not $[F(1,11)=22.52, p<.01]$. No other main effect or interaction was significant. Latencies were generally longer in the color singleton block, again indicating that search was more difficult when the target and distractor colors switched unpredictably. An ANOVA with trial type as a single factor (baseline, onset, and noonset) showed that the latencies did not differ between the three trial types. Note that in all three blocks, the distractordirected saccades had shorter latencies than the correct target-directed eye movements, particularly those elicited by abrupt onsets.

For both accuracy and latency, the two constant target blocks replicated the results from our previous study. That is, goal-driven saccade programming was disrupted by abrupt onset distractors. When observers knew the target and distractor colors beforehand, the disruptive effect of similar (onset) distractors was much larger than that of dissimilar (onset) distractors. Dissimilar onsets

Table 2

Mean Latencies (in Milliseconds) of First Saccades to the Target and Additional Distractor in the Three Blocks of Experiment 1

\begin{tabular}{cccccc}
\hline & \multicolumn{5}{c}{ Trial Type } \\
\cline { 2 - 6 } Saccade & Baseline & $\begin{array}{c}\text { Similar } \\
\text { Onset }\end{array}$ & $\begin{array}{c}\text { Similar } \\
\text { No-Onset }\end{array}$ & $\begin{array}{c}\text { Dissimilar } \\
\text { Onset }\end{array}$ & $\begin{array}{c}\text { Dissimilar } \\
\text { No-Onset }\end{array}$ \\
\hline Red target & 238 & 289 & 288 & 245 & 240 \\
Distractor & - & 217 & 250 & 195 & 269 \\
Green target & 263 & 289 & 297 & 261 & 289 \\
Distractor & - & 241 & 307 & 152 & 239 \\
Singleton target & 291 & - & - & 286 & 221 \\
Distractor & - & - & - & 221
\end{tabular}

Note-No data were collected for cells containing a dash; blank cells indicate that a particular event did not occur. 
did capture the eyes more frequently when observers were searching for a color singleton, but the interference was still much smaller than that from similar onset distractors in the constant target blocks. Therefore, it seems unlikely that the effect of target similarity observed in previous experiments and in the constant target blocks of this experiment was completely due to inhibition of all elements in the distractor color. The evidence from this experiment shows that the contribution of this topdown strategy was small.

\section{EXPERIMENT 2}

As noted, another explanation for the modulation by target similarity is that observers responded to local color discontinuities. The computation of visual salience based on local feature contrasts is incorporated in several models of visual search (Itti \& Koch, 2000; Li, 2002; Wolfe, 1994). In this experiment, the contribution of these local color discontinuities was explored by presenting blue distractors that were dissimilar from the red target and the remaining green distractors. These distractors created a color discontinuity that could not be used to select the target.

\section{Method}

Participants. Twelve new observers (4 men and 8 women; age range, 19-27 years) were recruited. All had normal or corrected-tonormal vision and were able to discriminate the colors used in the experiment.

Equipment and Stimuli. Search displays always consisted of five elements in this experiment: The baseline trials were dropped in favor of trials with an additional dissimilar blue distractor (CIE coordinates of .18/.20). These blue onset and no-onset distractors were of similar luminance as the placeholders and the other (red and green) search items.

Procedure. Observers were instructed to saccade to the red target that appeared among three green distractors. An additional distractor was present on every trial (equally often left and right). On half of the trials, this distractor had an abrupt onset. It could be (1) similar to the target, (2) dissimilar from the target but similar to the other distractors (green), or (3) different from the target and the distractors (blue). These three types of distractors were equally distributed over onset and no-onset trials. All different trial types were intermixed randomly. Participants performed two blocks of 154 trials, the first 10 of which were practice.

\section{Results and Discussion}

Data were handled in the same way as the constant target blocks were handled in the previous experiment. Thus, trials were rejected as a result of improper fixation $(3.91 \%)$, anticipatory saccades $(1.39 \%)$, or too small or too large an amplitude $(6.31 \%)$. In addition, for 1 participant the last 10 trials of one block were lost due to computer malfunction. Only a small number of saccades landed in an empty region or on a distractor other than the additional one (1.05\%). As before, these were excluded from further analyses.

Table 3 gives the percentage of first saccades to the additional distractor for all six trial types. An ANOVA with onset and similarity (three levels: similar, dissimilar green, and dissimilar blue) revealed main effects of both factors $[F(1,11)=63.02, p<.01$, and $F(2,22)=$ $235.24, p<.001$, respectively]. Again, the interaction between the two factors was significant $[F(2,22)=7.87$, $p<.05]$, indicating that the difference between onset and no-onset distractors varied over the three levels of similarity. Similar distractors (pooled over onset/no-onset) were more disruptive than dissimilar green and blue ones $[T(12)=0.00, p<.01$, for both comparisons $]$. Dissimilar blue distractors interfered slightly more with saccade programming than did dissimilar green ones $[T(12)=$ $9.00, p<.1]$. The results of these comparisons are identical when only onset distractors are taken into account.

The finding that the blue distractors were more disruptive than their green counterparts may be taken as evidence for the contribution of a set for color discontinuities. In addition, one might explain this finding in terms of top-down inhibition of the green items. However, the blue distractors did not capture the eyes nearly as often as the similar distractors, suggesting that the contribution of these strategies was very small. Thus, it appears that saccades were directed to the similar distractor because it shared its color with the target. The mean latencies of target and distractor-directed saccades are also listed in Table 3. As in the constant target blocks of Ex-

Table 3

Mean Percentage of Distractor-Directed Saccades and the Mean Latencies (in Milliseconds) of Both Target and Distractor-Directed Saccades in Experiment 2

\begin{tabular}{cccccc}
\hline $\begin{array}{c}\text { Similar } \\
\text { Onset }\end{array}$ & $\begin{array}{c}\text { Similar } \\
\text { No-Onset }\end{array}$ & $\begin{array}{c}\text { Dissimilar } \\
\text { Green Onset }\end{array}$ & $\begin{array}{c}\text { Dissimilar } \\
\text { Green No-Onset }\end{array}$ & $\begin{array}{c}\text { Dissimilar } \\
\text { Blue Onset }\end{array}$ & $\begin{array}{c}\text { Dissimilar } \\
\text { Blue No-Onset }\end{array}$ \\
\hline 56 & 29 & 3 & Percentage Errors & 5 & 1 \\
251 & 244 & 215 & Mean Correct SRT & 215 & 219 \\
197 & 233 & 196 & Mean Incorrect SRT & 221 & 269 \\
\hline
\end{tabular}

Note-Blank cells indicate that a particular event did not occur. 
periment 1 , target-directed saccades were initiated more slowly in the presence of a similar distractor, regardless of whether it had an abrupt onset $[F(2,22)=30.62, p<$ $.001]$. Saccades to the additional distractors generally had shorter latencies than the correct, target-directed ones.

Theeuwes et al. (1999) reported that the majority of fixation durations on the abrupt onset distractor were shorter than $150 \mathrm{msec}$. Because this interval would be too short to program a target-directed saccade "from scratch," the authors argued that the stimulus-driven saccade to the onset and the goal-driven saccade to the target were programmed in parallel. To examine the extent to which parallel programming occurred in the present study, vincentized (Ratcliff, 1979) cumulative frequency distributions of the fixation durations on the similar onset and similar no-onset distractors were calculated. Only those observers who fixated each of these distractors at least 10 times before redirecting gaze to the target contributed to this analysis. This was the case for all 12 participants on similar onset trials, giving a total of 254 trials; 7 observers fixated the similar no-onset distractor at least 10 times, resulting in a total of 106 trials.

The dashed lines in Figure 2 represent the two distributions. The median fixation durations on similar onset and similar no-onset distractors were 164 and $140 \mathrm{msec}$, respectively. Clearly, the fixation durations we observed were generally longer than in the Theeuwes et al. (1998; Theeuwes et al., 1999) studies, but still suggest that the distractor-directed and target-directed saccades were programmed in parallel on a proportion of trials. This appeared to be the case regardless of whether it is the onset or no-onset distractor that is fixated.

Figure 2 also illustrates the vincentized cumulative frequency distributions of the latencies of saccades to the similar onset and similar no-onset distractors (these distributions will be discussed in greater detail below).
The rank ordering of the four distributions suggests an interesting interdependence: Saccades to similar onsets are generally initiated faster than those to the no-onset distractors (difference of $29 \mathrm{msec}$ in the median latencies), but the subsequent target-directed saccade is then delayed by almost the same amount (difference of $24 \mathrm{msec}$ in the median fixation durations, in the opposite direction). It seems that the longer the erroneous saccade is delayed, the more time there is for the programming of the target-directed saccade, which, as a result, can then be initiated more quickly after fixating the distractor.

To see whether this relationship held on a trial-by-trial basis for individual observers, the correlation between the incorrect latency and subsequent fixation duration was calculated for each observer pooled over error type. ${ }^{2}$ The rank order of the distributions in Figure 2 would suggest a negative correlation between incorrect SRT and subsequent fixation duration. Indeed 11 out of 12 participants showed a negative correlation (ranging between -0.03 and -0.64$)$, and the mean value of -0.21 was significantly different from $0[t(11)=-2.69, p<$ $.05]$. The results from this correlational analysis provide additional evidence for the parallel programming of the distractor-directed and target-directed saccades: When the erroneous movement is delayed, the subsequent, correct saccade to the target can be initiated more quickly. Because this relation held regardless of error type, our analysis suggests the possibility of parallel programming of goal-driven, or endogenous, saccades.

\section{GENERAL DISCUSSION}

Both experiments replicated the basic pattern of performance observed in our previous study (Ludwig \& Gilchrist, 2002). That is, goal-driven saccade programming to a no-onset color target was disrupted by irrelevant

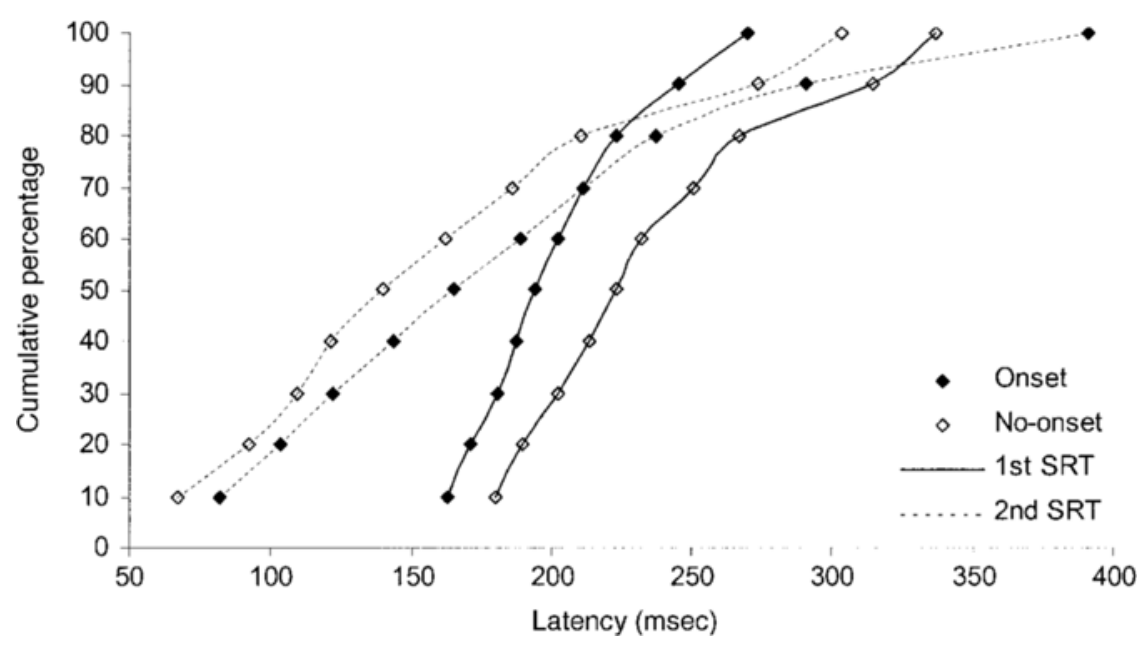

Figure 2. Vincentized (see text for details) cumulative frequency distributions of the (1) latency of erroneous saccades to (solid lines) and (2) the fixation durations on (dashed lines) similar onset $(N=254)$ and similar no-onset $(N=106)$ distractors in Experiment 2. 
onsets, particularly when these shared the target color. In this previous study, the target was red in every experiment, and red (similar) distractors interfered more with saccade programming than green (dissimilar) distractors. The two constant target blocks of Experiment 1 showed that this particular pattern was not due to something inherent in the red distractors that made them particularly disruptive (e.g., subtle luminance differences): Green onsets interfered more than red ones when observers were looking for a green target. Thus, it seems to be the target similarity that modulates oculomotor capture.

Here we proposed a number of top-down strategies that could have caused this modulation. One such strategy was the inhibition of all items in the distractor color, which would attenuate capture by dissimilar onsets. A condition in which the target was a color singleton indicated that the contribution of such a mechanism was small. Although dissimilar onsets were slightly more disruptive in this condition than when the target and distractor colors were known beforehand, the interference was still much less than that of similar onsets in the constant target blocks. Experiment 2 examined whether the similar distractors were more disruptive than dissimilar ones because they created a local color discontinuity, enhancing capture by similar onsets. Blue distractors that were dissimilar from the target, yet did create a color discontinuity, interfered more with saccade programming than did dissimilar green distractors, but the difference was very small. Taken together, these results demonstrate that stimulus-driven oculomotor capture by irrelevant onsets was genuinely modulated by target similarity. This adds strength to the conclusions of Ludwig and Gilchrist (2002) concerning the contingent capture hypothesis and models of saccade target selection. These points will be discussed in more detail below.

\section{Contingent Capture}

In line with the attentional cuing studies of Folk et al. (1992) and Folk et al. (1994), our experiments have shown top-down control settings to be a very powerful mediator of capture. That is, capture was more likely to occur when the additional distractor shared the feature (i.e., color) that defined the target. However, according to the contingent capture hypothesis, whether or not the distractor is presented with an abrupt onset should not matter, given that the target (display) was not defined by luminance transients. Clearly, this claim is not tenable in light of the present results.

However, in the present experiments, the search display itself was defined by the color change of the placeholders, and even though the physical luminance of the placeholder and search items was matched very closely, there may have been small perceptual luminance changes associated with the presentation of the search display. Therefore, it remains possible that observers adopted a set for transients in general, or for color and luminance transients specifically (Gibson \& Kelsey, 1998). Our re- sults are, in principle, consistent with such a weaker version of the contingent capture hypothesis.

In both experiments reported here, and in those of Ludwig and Gilchrist (2002), only similar distractors interfered with the saccadic latencies, regardless of whether or not they had an abrupt onset. One possible reason the onset component of the additional distractor did not interfere with the saccadic latencies is that in a search task such as the one used here, SRTs are too long to be influenced by an irrelevant luminance transient (Reingold \& Stampe, 2002). That is, it may be that there is a restricted time window within which onsets can delay movement initiation. The similarity effect is presumably dependent upon color processing in the ventral extrastriate areas, which is likely to be slower than the processing of a luminance onset (Barbur, Wolf, \& Lennie, 1998; Schmolesky et al., 1998). Therefore, the color-based similarity effect may take longer to develop (Ludwig \& Gilchrist, 2003), and exert a more sustained influence on the response latencies.

\section{Saccade Programming: Integration Versus Mixture Hypothesis}

The goal-driven modulation of capture by target similarity does not seem to accord with a strict dichotomy of stimulus-driven saccades mediated by a parieto-tectal route and goal-driven saccades dependent on the FEF (with its afferent projections from virtually all extrastriate areas). Because the predominantly magnocellular projections from V1/PEF into SC are not likely to convey color identity, it is hard to see why onsets in the target color are so much more disruptive than onsets of a similar luminance in any other color. Instead, our findings indicate that bottom-up signals (abrupt onsets) are combined with top-down target similarity information to jointly determine an item's salience. The idea that stimulus-driven and goal-driven signals converge onto a common oculomotor salience map is central to a recent computational model of saccade programming (Trappenberg, Dorris, Munoz, \& Klein, 2001) and will be referred to here as the "integration hypothesis" (see also Godijn \& Theeuwes, 2002).

Alternatively, as suggested by Ludwig and Gilchrist (2002), it may be that the entire population of erroneous saccades elicited by onsets in the target color consists of a group of genuinely stimulus-driven saccades triggered by the abrupt onset and a group of goal-driven saccades guided by the target color. To address this "mixture hypothesis," we examined the latency distributions of the erroneous saccades to similar onset and no-onset distractors. If the population of saccades to the similar onset distractor actually consisted of two subpopulations, one would predict a bimodal latency distribution: An early peak would represent a group of short-latency saccades triggered by the onset, and a later peak (overlapping with the distribution of saccades to similar no-onset distractors) would contain the saccades driven by the target color. 
These distributions are shown in Figure 2 (solid lines). Saccades to the similar onset distractors generally had shorter latencies than those to the similar no-onset distractors (medians of 194 and $223 \mathrm{msec}$, respectively). Figure 2 illustrates that this difference reflects a shift of the entire distribution and that it is not caused by a separate population of short-latency saccades to the similar onset. Although one has to be cautious in detecting the presence or absence of bimodality visually, particularly when dealing with vincentized distributions, this analysis of the error latencies can be taken as tentative evidence for the integration hypothesis. That is, saccades were directed to similar onset distractors because the combination of the luminance onset component and the color similarity component rendered these distractors more salient than similar no-onset or dissimilar onset distractors. It follows that the stimulus-driven onset signal is augmented by a goal-driven color similarity signal at a common site. It seems that saccades to abrupt onsets are not a fundamentally different type of eye movements than saccades to no-onset distractors or targets.

This notion runs counter to the widely held view that separate neural pathways are responsible for the programming of stimulus-driven and goal-driven saccades. There is now considerable neurophysiological support for the idea that both types of saccades share the same neural machinery. For instance, saccade-related FEF neurons increase their discharge before "reflexive" prosaccade errors in an antisaccade task (Everling \& Munoz, 2000). Moreover, the SC is involved in programming the correct, "voluntary" antisaccades (Everling, Dorris, Klein, \& Munoz, 1999). The contribution of the FEF is dependent on the intactness of the SC (Hanes \& Wurtz, 2001), and microstimulation of FEF saccade neurons activates cells in the SC coding the same movement vector (Schlag-Rey, Schlag, \& Dassonville, 1992). In addition, it is interesting to note that after monkeys were trained to search for a target of a constant color among distractors of another constant color, FEF visuomovement neurons seemed to develop color selectivity (Bichot, Schall, \& Thompson, 1996). That is, normally FEF neurons are not visually selective, and their activity evolves over time to indicate the location of a target among distractors. However, after training with a constant color target, FEF neurons signaled the target location very soon after presentation of the search array. In fact, this effect can already be seen when an oddball stimulus happens to be repeated on a number of trials (Bichot \& Schall, 2002). Such selectivity for items in the target color would allow for the interaction between fast onset signals and target similarity information in our experiments.

In summary, oculomotor capture can still be thought of as a race between a saccade program for the target and one for the distractor. However, this is not necessarily a competition between distinct types of saccades mediated by separate neural pathways. Our experiments indicate that the race is run within an integrated network of structures that are involved in eye movement programming.

\section{REFERENCES}

Bacon, W. F., \& Egeth, H. E. (1994). Overriding stimulus-driven attentional capture. Perception \& Psychophysics, 55, 485-496.

Barbur, J. L., Wolf, J., \& Lennie, P. (1998). Visual processing levels revealed by response latencies to changes in different visual attributes. Proceedings of the Royal Society of London: Series B, 265, 2321-2325.

Bichot, N. P., \& Schall, J. D. (2002). Priming in macaque frontal cortex during popout visual search: Feature-based facilitation and locationbased inhibition of return. Journal of Neuroscience, 22, 4675-4685.

Bichot, N. P., Schall, J. D., \& Thompson, K. G. (1996). Visual feature selectivity in frontal eye fields induced by experience in mature macaques. Nature, 381, 697-699.

Croner, L. J., \& Albright, T. D. (1999). Seeing the big picture: Integration of image cues in the primate visual system. Neuron, 24, 777-789.

Egeth, H. E., \& Yantis, S. (1997). Visual attention: Control, representation, and time course. Annual Review of Psychology, 48, 269-297.

Enns, J. T., Austen, E. L., Di Lollo, V., Rauschenberger, R., \& YANTIS, S. (2001). New objects dominate luminance transients in setting attentional priority. Journal of Experimental Psychology: Human Perception \& Performance, 27, 1287-1302.

Everling, S., Dorris, M. C., Klein, R. M., \& Munoz, D. P. (1999). Role of primate superior colliculus in preparation and execution of anti-saccades and pro-saccades. Journal of Neuroscience, 19, 27402754.

Everling, S., \& Munoz, D. P. (2000). Neuronal correlates for preparatory set associated with pro-saccades and anti-saccades in the primate frontal eye field. Journal of Neuroscience, 20, 387-400.

Folk, C. L., Remington, R. W., \& Johnston, J. C. (1992). Involuntary covert orienting is contingent on attentional control settings. Journal of Experimental Psychology: Human Perception \& Performance, 18, 1030-1044.

Folk, C. L., Remington, R. W., \& WRight, J. H. (1994). The structure of attentional control: Contingent attentional capture by apparent motion, abrupt onset, and color. Journal of Experimental Psychology: Human Perception \& Performance, 20, 317-329.

Gay mard, B., Ploner, C. J., Rivaud, S., Vermersch, A. I., \& PierrotDeseilligny, C. (1998). Cortical control of saccades. Experimental Brain Research, 123, 159-163.

Gibson, B. S., \& KelSEy, E. M. (1998). Stimulus-driven attentional capture is contingent on attentional set for displaywide visual features. Journal of Experimental Psychology: Human Perception \& Performance, 24, 699-706.

Godis, R. \& Theeuwes, J. (2002). Programming of endogenous and exogenous saccades: Evidence for a competitive integration model. Journal of Experimental Psychology: Human Perception \& Performance, 28, 1039-1054.

Guitton, D., Butchel, H. A., \& Douglas, R. M. (1985). Frontal lobe lesions in man cause difficulties in suppressing reflexive glances and in generating goal-directed saccades. Experimental Brain Research, 58, $455-472$.

Hanes, D. P., \& Wurtz, R. H. (2001). Interaction of the frontal eye field and superior colliculus for saccade generation. Journal of Neurophysiology, 85, 804-815.

Irwin, D. E., Colcombe, A. M., Kramer, A. F., \& Hahn, S. (2000). Attentional and oculomotor capture by onset, luminance and color singletons. Vision Research, 40, 1443-1458.

IтTI, L., \& KосH, C. (2000). A saliency-based search mechanism for overt and covert shifts of visual attention. Vision Research, 40, 1489-1506.

LI, Z P. (2002). A saliency map in primary visual cortex. Trends in Cognitive Sciences, 6, 9-16.

Ludwig, C. J. H., \& Gilchrist, I. D. (2002). Stimulus-driven and goaldriven control over visual selection. Journal of Experimental Psychology: Human Perception \& Performance, 28, 902-912.

Ludwig, C. J. H., \& Gilchrist, I. D. (2003). Target similarity affects saccade curvature away from irrelevant onsets. Experimental Brain Research, 152, 60-69.

Martin-Emerson, R., \& Kramer, A. F. (1997). Offset transients modulate attentional capture by sudden onsets. Perception \& Psychophysics, 59, 739-751. 
Miller, J. (1989). The control of attention by abrupt visual onsets and offsets. Perception \& Psychophysics, 45, 567-571.

Milligan, G. W. (1987). The use of the arc-sine transformation in the analysis of variance. Educational \& Psychological Measurement, 47, 563-573.

RATCLIFF, R. (1979). Group reaction time distributions and an analysis of distribution statistics. Psychological Bulletin, 86, 446-461.

Reingold, E. M., \& Stampe, D. M. (2002). Saccadic inhibition in voluntary and reflexive saccades. Journal of Cognitive Neuroscience, 14, 371-388.

SCHILlER, P. H. (1996). On the specificity of neurons and visual areas. Behavioural Brain Research, 76, 21-35.

Schlag-Rey, M., Schlag, J., \& Dassonville, P. (1992). How the frontal eye field can impose a saccade goal on superior colliculus neurons. Journal of Neurophysiology, 67, 1003-1005.

Schmolesky, M. T., Wang, Y. C., Hanes, D. P., Thompson, K. G., Leutgeb, S., Schall, J. D., \& Leventhal, A. G. (1998). Signal timing across the macaque visual system. Journal of Neurophysiology, 79, 3272-3278.

Theeuwes, J. (1994). Stimulus-driven capture and attentional set: Selective search for color and visual abrupt onsets. Journal of Experimental Psychology: Human Perception \& Performance, 20, 799-806.

Theeuwes, J., Kramer, A. F., Hahn, S., \& Irwin, D. E. (1998). Our eyes do not always go where we want them to go: Capture of the eyes by new objects. Psychological Science, 9, 379-385.

Theeuwes, J., Kramer, A. F., Hahn, S., Irwin, D. E., \& Zelinsky, G. J. (1999). Influence of attentional capture on oculomotor control. Journal of Experimental Psychology: Human Perception \& Performance, 25, 1595-1608.

Trappenberg, T. P., Dorris, M. C., Munoz, D. P., \& Klein, R. M. (2001). A model of saccade initiation based on the competitive integration of exogenous and endogenous signals in the superior colliculus. Journal of Cognitive Neuroscience, 13, 256-271.
Wolfe, J. M. (1994). Guided Search 2.0-A revised model of visual search. Psychonomic Bulletin \& Review, 1, 202-238.

YANTIS, S., \& JoNIDES, J. (1984). Abrupt visual onsets and selective attention: Evidence from visual search. Journal of Experimental Psychology: Human Perception \& Performance, 10, 601-620.

YANTIS, S., \& Jonides, J. (1990). Abrupt visual onsets and selective attention: Voluntary versus automatic allocation. Journal of Experimental Psychology: Human Perception \& Performance, 16, 121-134.

\section{NOTES}

1. The disappearance of the additional placeholder in the baseline and onset displays resulted in additional luminance transients that were not present in the no-onset trials. These luminance changes may influence the frequency of oculomotor capture. For instance, Martin-Emerson and Kramer (1997) and Miller (1989) demonstrated a reduction in attentional capture by onsets with a larger overall luminance change in the display. The influence of the additional offset was examined in the combined analysis of Experiments 1 and 2 of Ludwig and Gilchrist (2002). The additional placeholder offset did not affect the MRTs, and the amount of oculomotor capture was almost identical in displays with and without the offset.

2. As a first step, the individual correlations between SRT and fixation duration were calculated separately for saccades to the similar onset and to the similar no-onset distractors. Because the two sets of correlations did not differ significantly $[t(11)=0.34$, n.s. $]$ and because some of the correlations for the similar no-onset trials were based on a small number of observations, we pooled over error type.

(Manuscript received May 30, 2002; revision accepted for publication May 3, 2003.) 